\title{
Erratum zu: Therapie der akuten diabetischen Stoffwechselentgleisungen bei Erwachsenen (Update 2019)
}

\author{
Susanne Kaser · Harald Sourij · Martin Clodi · Bruno Schneeweiß · Anton N. Laggner · Anton Luger
}

Online publiziert: 6 . August 2019

(C) Der/die Autor(en) 2019

\section{Erratum zu:}

Wien Klin Wochenschr 2018

https://doi.org/10.1007/s00508-018-1423-z

Die Originalversion des Beitrags enthielt leider einen Fehler in Tab. 3. In der publizierten Version war in der 1. Spalte PO4 im Plasma mit 0 und Mg mit 1,25 angegeben, wobei auch Kalzium auf einen Wert im mittleren Bereich geändert werden soll. Der Autor entschuldigt sich für diesen Fehler.
Untenstehend die angepasste Tab. 3.

Open Access Dieser Artikel wird unter der Creative Commons Namensnennung 4.0 International Lizenz (http:// creativecommons.org/licenses/by/4.0/deed.de) veröffentlicht, welche die Nutzung, Vervielfältigung, Bearbeitung, Verbreitung und Wiedergabe in jeglichem Medium und Format erlaubt, sofern Sie den/die ursprünglichen Autor(en) und die Quelle ordnungsgemäß nennen, einen Link zur Creative Commons Lizenz beifügen und angeben, ob Änderungen vorgenommen wurden.

Tab. 3 Elektrolytkonzentrationen verschiedener Infusionslösungen (im Vergleich zu Plasma)

\begin{tabular}{|c|c|c|c|c|c|c|}
\hline & Plasma & $0,9 \% \mathrm{NaCl}$ & Elo-Mel ${ }^{\circledR}$ isoton & KADC & Ringer & Ringer-Laktat \\
\hline $\mathrm{Na}$ & 141 & 154 & 140 & 90 & 147 & 131 \\
\hline K & 4,5 & - & 5 & 25 & 4 & 5,4 \\
\hline $\mathrm{Ca}$ & 2,4 & - & 2,5 & 1,0 & 2,2 & 1,8 \\
\hline $\mathrm{PO}_{4}$ & 1,2 & - & - & 10 & - & - \\
\hline $\mathrm{Mg}$ & 0,8 & - & 1,5 & 1,5 & - & 1 \\
\hline $\mathrm{Cl}$ & 103 & 154 & 108 & 65 & 155 & 112 \\
\hline $\mathrm{HCO}_{3}$ & 24 & - & Acetat 45 & Malat 23 & - & Laktat 28 \\
\hline Osmolalität & $280-295$ & 308 & 302 & 215 & 309 & 278 \\
\hline
\end{tabular}

Die Online-Version des Originalartikels ist unter https:// doi. org/10.1007/s00508-018-1423-z zu finden.

\footnotetext{
S. Kaser

Department für Innere Medizin 1, Medizinische Universität Innsbruck, Innsbruck, Österreich

Christian Doppler Labor für Insulinresistenz, Medizinische Universität Innsbruck, Innsbruck, Österreich

H. Sourij

Klinische Abteilung für Endokrinologie und Diabetologie,

Universitätsklinik für Innere Medizin, Medizinische

Universität Graz, Graz, Österreich

M. Clodi

Abteilung für Innere Medizin, Krankenhaus Barmherzige

Brüder Linz, Linz, Österreich
}

\begin{abstract}
B. Schneeweiß
Abteilung für Innere Medizin, Landeskrankenhaus Kirchdorf, Kirchdorf, Österreich
\end{abstract}

A. N. Laggner

Universitätsklinik für Notfallmedizin, Medizinische

Universität Wien, Wien, Österreich
A. Luger $(\square)$
Klinische Abteilung für Endokrinologie und Stoffwechsel,
Universitätsklinik für Innere Medizin III, Medizinische
Universität Wien, Währinger Gürtel 18-20, 1090 Wien,
Österreich
anton.luger@meduniwien.ac.at 